\title{
Intrusion Detection and Defense Implementation (IDDI) in Cuckoo Hashing
}

\author{
D.Seethalakshmi ${ }^{1}$, Dr.G.M.Nasira ${ }^{2}$ \\ ${ }^{1}$ Research Scholar Research and Development Centre Bharathiar University, Coimbatore, Tamilnadu, India \\ ${ }^{2}$ Assistant Professor and Head Department of Computer Applications Chikkanna Government Arts College \\ Tirupur, Tamilnadu, India
}

\begin{abstract}
The cuckoo hashing methodology completely avoids hash collisions. An insertion of a new item causes a failure and an endless loop when there are collisions in all probed positions till achieving the timeout status. To interrupt the countless loops, an intuitive manner is to carry out a complete rehash if this uncommon incident takes place. In practice, the high priced overhead of acting a rehashing operation can be dramatically decreased by using taking benefit of a very small extra regular-size space. In our proposed paper hashing involve collisions in which the stored buckets with one item will be reduced to probe the hash collision and query about the buckets. The enumerator that allocates each hash bucket will reduce the endless loops. As an accession we include intrusion detection and defense implementation (IDDI) that scrutinizes the hash and cache memory utilization efficiently. Cuckoo hashing is more prone to intrusion attack for which we alleviate such attacks by avoiding loops in buckets. Through detection and avoidance mechanism of intrusion we enhance the transmission in cuckoo hashing simultaneously by providing strong security towards its data.
\end{abstract}

Keywords: Cloud computing Data, cuckoo hashing, Intrusion Detection and Defense Implementation (IDDI), enumerator

\section{Introduction}

The cuckoo hashing defined in dynamic static dictionary with hashing leverages or greater hash functions for managing hash collisions to mitigate the computing complexity of the use of the linked lists of traditional hash tables. In order to deal with these challenges, we recommend an enumerator scheme for cloud garage structures to mitigate the actual hash collisions and excessive latency in the insertion procedure [1]. Enumerator lets in each item to have candidate buckets. An empty bucket can be chosen to shop the item. The file kicking-out times taking place on the bucket in real time, we allocate a counter for each bucket [2]. When hash collisions occur for the duration of the insertion operation, and all candidate buckets aren't empty, the item selects the bucket with the minimal counter to kick out the occupied object to lessen or avoid infinite loops.

The motive of Enumerator is to choose kicking-out routes independently instead of randomly choose and searching for the empty buckets as fast as possible [3]. Furthermore, with a purpose to reduce the frequency of rehashing, we quickly keep insertion-failure gadgets into in-reminiscence cache, rather than directly rehash the entire structure. Alleviating Hash Collisions and statistics Migration we advocate a unique cuckoo hashing primarily based scheme, referred to as Enumerator, in the cloud garage servers.

Enumerator allocates a counter consistent with bucket of hash tables, to file the kicking-out instances taking place within the buckets [4]. Whilst candidate positions of a new item to be inserted are all occupied by other gadgets, Enumerator selects the minimum counter, rather than randomly pick out, to execute the replacement operation. This scheme can considerably alleviate hash collisions and decrease records migration by way of balancing gadgets in hash tables [5].

\section{Literature Survey}

In Cuckoo hashing R. Pagh and F. F. Rodler et al explains the appealing properties of cuckoo hashing in parallel representation. Particularly, no dynamic memory allocation is needed, updates are done in anticipated amortized steady time, and group queries are done in worst case steady time. Furthermore, with high chance, the research system queries and memory entries that is independent and can be queried in parallel [6]. The approach underlying our creation is to enforce a canonical reminiscence illustration on cuckoo hashing. This is, up to the preliminary randomness, each set of elements has a completely unique memory representation. Practical records-unbiased dynamic dictionary based on cuckoo hashing [7]. In a records-unbiased records structure, the reminiscence representation at any factor in time yields no statistics at the specific sequence of insertions and deletions that caused its contemporary content material, apart from the content material itself. This type of property is significant while stopping unintended leakage of information, and was also found beneficial in several algorithmic settings [8]. M. Zukowski, S. H'eman, and P. Boncz, et al in their architecture conscious hashing explains rapid hash features, ways to efficiently deal with multi-column keys and endorse the usage of a 
currently brought hashing scheme called Cuckoo Hashing over the normally used bucket-chained hashing [9]. Inside the second element of the paper, we awareness at the CPU cache utilization, through dynamically partitioning facts streams such that the partial hash tables in shape inside the CPU cache. Conventional partitioning works as a separate preparatory segment, forcing materialization, which can also require $\mathrm{I} / \mathrm{O}$ if the movement does no longer match in RAM [10]. We introduce quality-effort partitioning, a technique that interleaves partitioning with execution of hash-based totally question processing operators and avoids me I/O. Inside the system they found a way to prevent issues in partitioning with cache line alignment, which can strongly decrease throughput [11].

O. Shalev and N. Shavit et al in Split-ordered lists: Lock-free extensible hash tables the new mathematical shape on the center of their set of rules is recursive split ordering, a manner of ordering factors in a connected list so that they may be again and again "break up" the use of a single evaluate-and-swap operation [12]. The prior recognized algorithms in that extensibility is derived via "moving the buckets most of the objects" instead of "the items some of the buckets [13]." even though lock-based algorithms are anticipated to work first-rate in multi-programmed environments, empirical tests we conducted on a huge shared reminiscence multiprocessor display that even in non-multi-programmed environments, the brand new algorithm performs as well as the maximum efficient known lock-based totally resizable hash-desk algorithm, and in excessive load instances it extensively outperforms it [14].

X. Li, D. G. Andersen, M. Kaminsky, and M. J. Freedman et al in their Algorithmic improvements for fast concurrent cuckoo hashing fast concurrent hash tables are an increasingly more important building block as we scale structures to greater numbers of cores and threads [15]. This paper offers the design, implementation, and evaluation of a excessive-throughput and reminiscence-green concurrent hash table that helps a couple of readers and writers. The layout arises from cautious interest to structures-degree optimizations which include minimizing important section period and reducing inter processor coherence traffic through algorithm reengineering [16]. As a part of the architectural foundation for this engineering, we encompass a dialogue of our experience and outcomes adopting Intel's recent hardware transactional memory (HTM) aid to this crucial constructing block [17].

\section{Conventional Cuckoo Hashing Standards}

The cuckoo hashing is a dynamic static dictionary and helps rapid queries with the worst-case steadyscale lookup time due to flat addressing for an object among more than one choice. Cuckoo hashing gives a couple of, no longer one, hash positions for an item and permits the gadgets to transport inside these hash positions [18]. Moreover, the gap overhead is about second space gadgets, that's just like the gap overhead of binary search trees. Hash tables to illustrate the practical operations of widespread cuckoo hashing. We use arrows to reveal feasible locations for shifting items [19]. If item $\mathrm{x}$ is inserted into hash tables, we first test whether or not there exists any empty bucket of candidates of object $\mathrm{x}$. If no longer, we randomly pick one from applicants and kick out the authentic item [20]. The kicked-out item is inserted into Table in the equal way.

The manner is accomplished in an iterative manner, till all objects discover their buckets [21]. The demonstration of the running method that the object $\mathrm{x}$ is correctly inserted into the Table by way of moving items $\mathrm{a}$ and $\mathrm{b}$ from one table to the opposite. At the same time as, as proven with endless loops may arise and some items fail to discover appropriate bucket to be stored [22]. Therefore, a threshold "MaxLoop" is important to specify the range of iterations. If the generation instances are same to the pre-defined threshold, we will argue the occurrence of endless loops, which reasons the re-construction of entire shape. The theoretical evaluation of MaxLoop with vital property of random desire in hash functions, hash collisions can't be absolutely prevented, however extensively alleviated. It's important to correctly guide concurrent access to a cuckoo hash desk. Some previously proposed schemes are used to enhance concurrency. So one can help arbitration for concurrent get admission to in records structures, locking are an effective mechanism.

\section{Intrusion Detection And Defense}

Multi-thread programs gain high overall performance via taking advantage of an increasing number of cores. That allows you to ensure thread correctness and safety, a vital phase managed by means of a lock is used to permit the operations of a couple of threads to be serialized when gaining access to the shared part of information. It's far hard to correctly aid concurrent access to cuckoo hash tables. On the way to guide the concurrent execution of the unmarried-thread cuckoo hashing algorithm, while still keeping the high space efficiency of cuckoo hashing, we want to cope with two issues.

Intrusion detection is the technique of monitoring the occasions taking place in a computer system or network and studying them for signs of viable incidents, which are violations or forthcoming threats of violation of data transmission safety policies, appropriate use rules, or modern hashing protection practices. Intrusion prevention is the system of acting intrusion detection and trying to stop detected feasible incidents. 
Intrusion detection and prevention systems are frequently targeted on figuring out feasible incidents, logging records approximately them, trying to stop them, and reporting them to protection in cuckoo hashing. Similarly, companies use Intrusion detection for other purposes, which include identifying troubles with safety rules, documenting current threats and deterring individuals from violating security policies. Intrusion detection system has grown to be a important addition to the safety infrastructure of nearly each employer. Intrusion detection normally hash file detection associated with located events, notify safety directors of important determined activities, and produce reviews. Many intrusion preventions can also reply to a detected danger through trying to prevent it from succeeding. They use several response techniques, which contains hash collision attacks stopping the attack itself, changing the security surroundings or converting the attack in hash buckets. Thus we design, implement, configure, secure, tracks, and defense cuckoo hashing methodologies. The kinds of IDPS technology are differentiated frequently through the kinds of events that they reveal and the approaches in which they're deployed. Therefore, it's far critical for them to cost the enhancements added by these new system.

\section{Experimental And Performance Analysis}

Intrusion Defense structures are taken into consideration extensions of Intrusion Detection systems because each system reveals network visitors and/or gadget prone for threats. The number one difference among the two systems is that Intrusion defense systems are positioned in-line and are consequently able to actively save you/block intrusions which are detected. Mainly, an intrusion detection and defense system can take such movements as sending an alarm, losing malicious packets, resetting the relationship and/or blocking off site visitors from an offending IP address. An IPS can also correct Cyclic Redundancy test (CRC) errors, defragment packet streams, prevent TCP sequencing troubles, and clean up undesirable shipping and community layer alternatives.

In the below message transmission of hashing buckets the message packet streams can be layered and transmitted accordingly as follows,

$$
\alpha=\beta-\gamma
$$

Where $\alpha$ seems to be the retained messages for which the total number of incoming messages $(\beta)$ will be deducted with the total number of outgoing messages $(\gamma)$. Intruders know how signature-primarily based detection works, and in response they've evolved a number of ways of evading detection, typically by means of introducing diffused variations of their attacks. For this reason, main IPS makers normally post vulnerabilitybased totally guidelines (in preference to exploit-based totally signatures) to hit upon all feasible variants of an assault. They may additionally offer anomaly-primarily based detection strategies, that detects the attack which termed as

$$
\delta=\alpha>\theta
$$

From the above the attack virus $(\delta)$ will be detected as the greater than normal $(\theta)$ will be the message to be retained $(\alpha)$. The intruder (l) will be determined by the lesser than value in comparing with normal values of the cache clearance $(c l)$ and accidental loss $(a l)$.

$$
l=\alpha<\theta
$$

Thus from the above equations the algorithm defined to be as

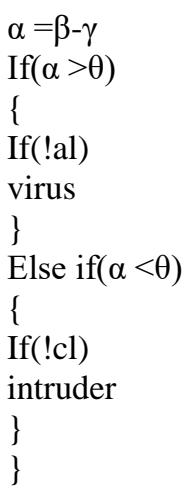

The intrusion attacks to be detected in prior will be provided defense from the hashing buckets. In Intrusion prevention might also study the series of events while the user of a web utility logs in, and after logging in issues instructions to the utility to carry out design. The IPS may recall a person issuing instructions without logging in to be an event that should be blocked, due to the fact this will be a sign of an outsider who is making an attempt to carry out unauthorized transactions. 


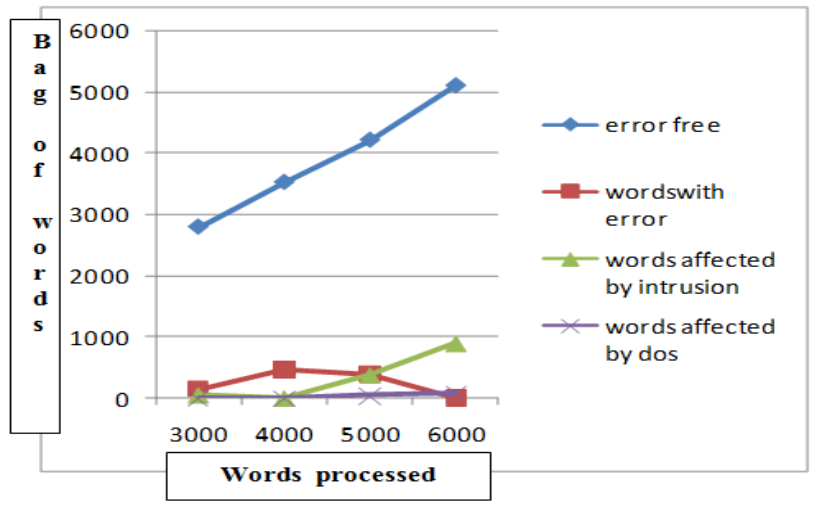

Figure.1 Graph Representation for comparing processed words along with bag of words

The above graph depicts the number of words processed along with bag of words for error free intrusion detection. The hassle with host-based totally detection is that the attacker, once he has been capable of compromise a device, may be in a position to observe the presence of HIDS or HIPS on the machine. Frequently goal structures that keep transmit or records. So it might make experience that a number-based totally detection and prevention. That allows you to detect assaults on systems, community-based IDSs and IPSs offer a key benefit over host-based totally answers. The major reason for this is that the intruder will now not be capable of examine any of the detection/prevention talents.

\section{Conclusion}

In our paper we propose intrusion detection and defense system for hashing, at the same time as more hard to come across than ordinary malware, can often be detected, furnished the enterprise is inclined to invest within the gear required to repel them. Intrusion detection at the other hand correlates threats against endpoint intelligence to lessen the quantity of "actionable" security. Thus our system provided agility, rapid deployment, improvising the system availability and defense from vulnerable detections. In future we enhance the cuckoo hashing detection system by providing defense mechanism to other vulnerable attacks too.

\section{References}

[1] W.-C. Chen and J. S. Vitter, “Analysis of new variants of coalesced hashing,” Proc. TODS, vol. 9, no. 4, pp. 616-645, 1984.

[2] J. S. Vitter and W.-C. Chen, "The design and analysis of coalesced hashing". Oxford University Press, Inc., 1987.

[3] R. Pagh and F. F. Rodler, Cuckoo hashing. Springer Berlin Heidelberg, 2001.

[4] M. Zukowski, S. H'eman, and P. Boncz, "Architecture-conscious hashing," workshop on Data management on new hardware, 2006.

[5] M. M. Michael, "High performance dynamic lock-free hash tables and list-based sets," Parallel algorithms and architectures, pp. 73-82, 2002.

[6] O. Shalev and N. Shavit, "Split-ordered lists: Lock-free extensible hash tables," Proc. JACM, vol. 53, no. 3, pp. 379-405, 2006.

[7] J. Triplett, P. E. McKenney, and J. Walpole, "Scalable concurrent hash tables via relativistic programming," ACM SIGOPS Operating Systems Review, vol. 44, no. 3, pp. 102-109, 2010.

[8] J. Triplett, P. E. McKenney, and J. Walpole, "Resizable, scalable, concurrent hash tables via relativistic programming," Proc. USENIX ATC, 2011.

[9] B. Fan, D. G. Andersen, and M. Kaminsky, "Memc3: Compact and concurrent mem cache with dumber caching and smarter hashing" Proc. USENIX NSDI, vol. 13, pp. 385-398, 2013.

[10] X. Li, D. G. Andersen, M. Kaminsky, and M. J. Freedman, "Algorithmic improvements for fast concurrent cuckoo hashing," Proc. Euro Sys, 2014.

[11] D. Fotakis, R. Pagh, P. Sanders, and P. Spirakis, "Space efficient hash tables with worst case constant access time," Proc. STACS, pp. 271-282, 2003.

[12] A. Frieze, P. Melsted, and M. Mitzenmacher, "An analysis of random walk cuckoo hashing," Approximation, Randomization, and Combinatorial Optimization. Algorithms and Techniques, pp. 490-503, 2009.

[13] B. K. Debnath, S. Sengupta, and J. Li, "Chunkstash: Speeding up inline storage deduplication using flash memory.," Proc. USENIX ATC, 2010.

[14] A. Kirsch, M. Mitzenmacher, and U. Wieder, "More robust hashing: Cuckoo hashing with a stash," Proc. SICOMP, vol. 39, no. 4, pp. $1543-1561,2009$.

[15] R. Pagh and F. F. Rodler, "Cuckoo hashing," Journal of Algorithms, vol. 51, no. 2, pp. 122-144, 2004.

[16] R. Pagh, "On the cell probe complexity of membership and perfect hashing," Proc. STOC, pp. 425-432, 2001.

[17] Y. Hua, B. Xiao, and X. Liu, "Nest: Locality-aware approximate query service for cloud computing," Proc. INFOCOM, pp. 1327$1335,2013$.

[18] Y. Hua, B. Xiao, X. Liu, and D. Feng, "The design and implementations of locality-aware approximate queries in hybrid storage systems," Proc. TPDS, vol. 26, no. 11, pp. 3194-3207, 2015.

[19] Y. Hua, H. Jiang, and D. Feng, "Fast: near real-time searchable data analytics for the cloud," Proc. SC, pp. 754-765, 2014.

[20] B. Debnath, S. Sengupta, and J. Li, "Flashstore: high throughput persistent key-value store," VLDB Endowment, vol. 3, no. 1-2, pp. 1414-1425, 2010.

[21] M. Herlihy and N. Shavit, "The art of multiprocessor programming," Proc. PODC, vol. 6, pp. 1-2, 2006.

[22] B. Fitzpatrick and A. Vorobey, "Memcached: a distributed memory object caching system," 2011. 